\title{
Should knee extension strength testing be implemented as a screening test for identifying probable and confirmed sarcopenia in older T2DM patients?
}

Ofer kis ${ }^{1 *}$, Assaf Buch ${ }^{2,3,4} \mathbb{D}$, Roy Eldor ${ }^{2}$, Amir Rubin $^{5}$, Ayelet Dunsky ${ }^{5}$, Naftali Stern ${ }^{3,6}$ and Daniel S. Moran ${ }^{1}$

\begin{abstract}
Background: The accelerated loss of muscle strength and mass observed in older type 2 diabetes mellitus (T2DM) patients due to the combined effects of diabetes and obesity, greatly increases their risk for sarcopenia. Early detection and treatment of probable and confirmed sarcopenia is paramount to delay mobility disability. Using low handgrip strength cut-off points for the initial identification of sarcopenia according to the new European Working Group on Sarcopenia in Older People (EWGSOP2) guidelines may mask the presence of sarcopenia. Relative knee extension strength cut-off points using a simple hand-held dynamometer can assist clinicians in the diagnosis of probable and confirmed sarcopenia by possibly reducing false negative results.
\end{abstract}

Methods: A cohort of one hundred T2DM older patients (60\% women) (mean age 74.5 years) mostly obese community dwelling older adults were evaluated for body composition by Bioelectrical impedance analysis (BIA), yielding appendicular skeletal mass index (ASMI) results. Patients underwent handgrip strength (HGS) and knee extension strength (KES) tests as well as functional ability tests. Prevalence of probable and confirmed sarcopenia using HGS and KES cut-off points were calculated. Pearson correlations were performed to evaluate the relationship between ASMI and limbs strength. A regression analysis was conducted to examine which variables best predict ASMI values. A multivariate analysis of covariance was performed to assess the effect of independent variables on KES and HGS.

Results: Using cutoff points for low KES identified 24 patients with probable sarcopenia and two with confirmed sarcopenia. Conversely, using the EWGSOP2 cut off points for low HGS, identified only one patient with probable sarcopenia and none of the patients with confirmed sarcopenia.

Conclusion: KES cut-off points using a simple hand-held dynamometer can assist in the identification of probable and confirmed sarcopenia using EWGSOP2 cut off points for low muscle mass in a population of older T2DM patients for further analysis and early treatment. This is notably true in patients possessing high body mass index (BMI) alongside normal ASMI and HGS, potentially reducing false positive sarcopenia screening results.

\footnotetext{
* Correspondence: oferkis58@gmail.com

'The Faculty of Health Sciences, Ariel University, Ariel, Israel

Full list of author information is available at the end of the article
} 
Trial registration: ClinicalTrials.gov PRS: NCT03560375. Last registration date (last update): 06/06/2018. The trial was a-priori registered before actual recruitment of subjects.

Keywords: Appendicular skeletal mass index, Handgrip strength, Older adults, Knee extension strength, Sarcopenia, Type 2 diabetes

\section{Introduction}

The prevalence of diabetes in the US is steadily increasing, reaching $26.8 \%$ ( $14 \%$ in men and $12 \%$ in women) among adults aged 65 years or older [1]. The estimated global diabetes prevalence is $19.3 \%$, with North America and the Caribbean possessing the highest regional diabetes prevalence of 27\% [2]. Type 2 diabetes mellitus (T2DM) is associated with an accelerated reduction in muscle mass [3], strength [4] function [5], disability [6] and frailty [7], resulting in reduced autonomy and an increased burden on public health care systems [8].

Sarcopenia, characterized by a progressive loss of skeletal muscle mass, strength, and functional abilities [9] is a common complication in older patients with T2DM $[10,11]$, further increasing their risk for functional decline [12], and physical disability [13]. Compared to the general older population, studies evaluating sarcopenia in older T2DM patients are scarce with prevalence rates varying greatly, ranging from 7 to $29.3 \%$ [14]. This disparity is attributed mainly to variations between populations, variations in quantitative evaluation methods, as well as different diagnostic criteria [14, 15].

The European Working Group on Sarcopenia in Older People (EWGSOP2) recently updated its original algorithm for "sarcopenia case-finding". Muscle strength rather than muscle mass has become the main criteria for diagnosis termed "probable sarcopenia" as muscle strength is the most reliable measure of muscle function [16]. Sarcopenia is further confirmed by the additional detection of a low muscle mass measurement. The EWGSOP advises the use of either the chair rise test, but preferably the use of a handgrip strength test (HGS), due to it its association with functional limitations, and ease of administration in clinical settings.

Older patients with T2DM typically experience a significant loss of lower body strength [17-19] which is associated with a deteriorating health status [20], impaired mobility [21] and loss of autonomy [22]. Isometric knee extension strength testing (KES) using a relatively inexpensive hand-held dynamometer has been found to be valid and reliable in assessing lower body muscle strength with a moderate to high correlation to isokinetic measurements (considered to be the gold standard method) [23-25],especially in older populations [26]. Furthermore, KES has been found to be superior to HGS as an indicator of muscle dysfunction in patients with T2DM [27]. Due to the increased adiposity often accompanying T2DM older patients, it is recommended that knee extension strength relative to bodyweight should be measured as it better relates to low mobility than absolute strength scores [28].

Due to the importance in the early detection and treatment of probable [29] and confirmed sarcopenia, we aim to identify the most appropriate screening strength test by comparing the prevalence of sarcopenia by the use of relative KES to absolute HGS measurements in older patients with T2DM. To this end, we evaluated the association between KES and HGS to appendicular skeletal mass index (ASMI), and the prevalence of low-test scores of sarcopenia parameters and common physical performance screening tests.

\section{Methods}

\section{Study design}

The present investigation is an analysis of baseline measurements of patients enrolled in a randomized clinical trial, which investigated the efficacy of resistance strength training, pharmacotherapy (empagliflozin), and a diet intervention (vegetarian), on the prevention of sarcopenia and/or frailty [30]. The analysis presented here in, is a cross-sectional analysis.

\section{Subjects' characteristics}

Overall, 100 older ( $\geq 65$ years) male and female T2DM patients, diagnosed in accordance with the American Diabetes Association guidelines were recruited in the original study and their baseline data is presented in the current study (Table 1). The subjects were patients of the various out-patient clinics at the institute of Endocrinology, Metabolism, and Hypertension (IEMH), Tel-Aviv Sourasky Medical Center (TASMC). Eligible patients passed a physician interview and physical examination. A comprehensive survey which included health status, lifestyle as well as physical activity habits were filled in by the patients. The main Inclusion criteria included: Performing $\leq 2$ days a week of any leisure aerobic physical activity, walking independently either with or without an assistance device and $\mathrm{HbA} 1 \mathrm{C} \geq 6.5 \%$ to $\leq 8 \%$. Exclusion criteria included: performing any resistance training within the past six months, the use of anabolic or catabolic steroid agents, severe peripheral neuropathy, end-stage renal failure, history of stroke, myopathy, motor functional disorders and treatment with SGLT-2 inhibitors. The recruitment process as well as the complete eligibility criteria are described in detail elsewhere [30]. 
Table 1 Participants characteristics

\begin{tabular}{|c|c|c|c|}
\hline Variables & Men $(n=40)$ & Women $(n=60)$ & Total $(n=100)$ \\
\hline \multicolumn{4}{|l|}{ Anthropometric measurements } \\
\hline Age (years) & $70.5 \pm 4.2$ & $70.4 \pm 5.0$ & $70.5 \pm 4.6$ \\
\hline height (cm) & $172 \pm 6.1$ & $158 \pm 4.5$ & $1.63 \pm 0.1$ \\
\hline Weight (kg) & $94.8 \pm 16.4$ & $78.3 \pm 14.2$ & $84.7 \pm 17.3$ \\
\hline WC (cm) & $113.5 \pm 13.7$ & $106.6 \pm 13.6$ & $109.2 \pm 14$ \\
\hline Body Mass Index (kg/m2) & $31.1 \pm 5.6$ & $31.4 \pm 6.1$ & $31.6 \pm 5.9$ \\
\hline Lean body mass leg (kg) & $9.18 \pm 1.35$ & $6.3 \pm 0.87$ & $7.35 \pm 1.7$ \\
\hline Lean body mass arm (kg) & $3.64 \pm 0.53$ & $2.37 \pm 0.46$ & $2.84 \pm 0.7$ \\
\hline ASMI (kg/m2) & $8.55 \pm 0.9$ & $6.90 \pm 0.9$ & $7.52 \pm 1.2$ \\
\hline ASM (kg) & $25.3 \pm 3.36$ & $17.1 \pm 4.9$ & $20.36 \pm 4.2$ \\
\hline Fat mass (\%) & $36.4 \pm 6.7$ & $43.8 \pm 5.8$ & $40.8 \pm 6.2$ \\
\hline \multicolumn{4}{|l|}{ Strength and functional measurements } \\
\hline KES (kg) & $37.58 \pm 8.0$ & $24.0 \pm 5.6$ & $29.2 \pm 9.4$ \\
\hline KES/weight (kg/kg) & $0.39 \pm 0.07$ & $0.31 \pm 0.06$ & $0.34 \pm 0.1$ \\
\hline HGS (kg) & $40.06 \pm 6.83$ & $24.97 \pm 4.3$ & $31 \pm 9.2$ \\
\hline HGS/weight (kg/kg) & $0.44 \pm 0.1$ & $0.33 \pm 0.07$ & $0.37 \pm 0.1$ \\
\hline \multicolumn{4}{|l|}{ Clinical Data } \\
\hline Obesity: BMI > 30 (\%) & 65.0 & 55.0 & 59 \\
\hline Obesity: WC > $102 \mathrm{~cm}$ men (\%) > $88 \mathrm{~cm}$ women (\%) & 87.5 & 96.6 & 93 \\
\hline Obesity: > 1 SD than mean reference values of $\%$ fat (\%) ${ }^{1}$ & 77.5 & 65 & 70 \\
\hline Diabetes duration (years) & $12.3 \pm 10.1$ & $13.7 \pm 8.8$ & $13.37 \pm 9.4$ \\
\hline Diabetes> 10 years $(\%)$ & 58.7 & 67.8 & 64 \\
\hline $\mathrm{HbA1c}(\%)$ & $7.63 \pm 1.3$ & $7.34 \pm 0.9$ & $7.46 \pm 1.14$ \\
\hline Neuropathy (\%) & 12.5 & 17.0 & 15.2 \\
\hline Nephropathy (\%) & 15.0 & 6.8 & 10.1 \\
\hline Retinopathy (\%) & 7.7 & 8.8 & 8.3 \\
\hline IHD (\%) & 10.6 & 5.3 & 7.4 \\
\hline CHF (\%) & 5.2 & - & 2 \\
\hline Hypertension (\%) & 56.5 & 54.3 & 55.2 \\
\hline Statin use (\%) & 64.1 & 52.6 & 57.1 \\
\hline Polypharmacy (> 8 med's) & 25 & 30 & 28 \\
\hline Low vitamin $\mathrm{D}<25$ ng/mL (\%) & 43.3 & 43.6 & 43.4 \\
\hline \multicolumn{4}{|l|}{ Physical performance } \\
\hline Gait speed (m/s') & $1.09 \pm 0.2$ & $1.08 \pm 0.18$ & $1.08 \pm 0.19$ \\
\hline Timed up \& go $\left(s^{\prime}\right)$ & $10.6 \pm 1.7$ & $10.87 \pm 2.16$ & $10.76 \pm 2.0$ \\
\hline Chair stands $30 \mathrm{~s}^{\prime}$ & $11.28 \pm 1.8$ & $10.6 \pm 2.9$ & $10.99 \pm 2.35$ \\
\hline 2 Min' walk Test (m) & $172 \pm 25.9$ & $158.37 \pm 23.65$ & $163.7 \pm 25.3$ \\
\hline
\end{tabular}

Abbreviations: ASM Appendicular Skeletal Mass, ASMI Appendicular Skeletal Mass Index, BMI Body Mass Index, CHF Chronic Heart Failure, HGS Hand Grip Strength, IHD Ischemic Heart Disease, KES Knee Extension Strength, WC Waist Circumference

1-According to normative data published [35]

\section{Muscle quantity and anthropometry}

Body composition measurements were obtained through direct segmental multi-frequency bioelectrical impendence analysis (BIA) technique method (InBody 770 body composition analyzer, InBody Co., Ltd, Seoul,
Korea), by the same technician throughout the study. Measurements included body weight (BW), total and segmental (both legs, trunk, and both arms) skeletal muscle mass, fat mass and \% body fat. The In-Body 770 is a valid tool for the assessment of total body and 
segmental body composition [31]. Height was measured electronically to the nearest $0.1 \mathrm{~cm}$, and body mass index (BMI) was calculated. Waist circumference (WC) was measured using a designated tape measure. ASMI was calculated by adding the sum skeletal masses of both arms and legs divided by height squared using the BIA technique [31]. Low muscle mass was defined according to the EWGSOP2 as ASMI $<7.0 \mathrm{~kg} / \mathrm{m}^{2}$ in men and $<5.5$ $\mathrm{kg} / \mathrm{m}^{2}$ in women [16].

\section{Muscle strength assessment}

Upper body muscle strength was assessed by a handgrip strength test using a hand-held dynamometer Jamar $^{\circ}$ Sammons Preston Rolyan, Chicago, Illinois, USA). Subjects were seated in the upright position with the arm along their side, the elbow bent at $90^{\circ}$ with the arm supported horizontally by a tester and feet firmly planted on the floor. The width of the dynamometer handle was adjusted to fit the size of the hand (generally using the second smallest grip). One orientation trial was performed for each hand before three trials alternating between arms were performed. The highest of the six measurements was recorded [32]. All trials were separated by a pause of $60 \mathrm{~s}$. Low muscle strength was defined in accordance to the revised EWGSOP2 guidelines as HGS < $27 \mathrm{~kg}$ in men and $<16 \mathrm{~kg}$ in women [16].

Lower body muscle strength was assessed by an isometric KES test using a hand-held dynamometer (hydraulic Push-Pull Dynamometer Baseline ${ }^{\bullet}$ evaluation industries). KES tests using a hand-held dynamometer, have been found to possess both high interrater and intrarater reliability (ICC $\geq 0.95$ and $\mathrm{ICC}=0.948$, respectively) [33]. Participants were asked to sit with their lower legs over the end of a standard examination table, with hips and knees flexed to $90^{\circ}$, and to perform a maximal isometric contraction with the tester's encouragement. To increase the test's validity and reliability, the examiner stabilized himself against the subject's knee extension by positioning one knee on the floor and the other foot against a wall. The dynamometer was placed on the anterior part of the lower leg, just above the talotibial joint line, making sure the subject felt no pain, for maximum muscular contraction. A preliminary orientation test was performed on each leg prior to the execution of three $5 \mathrm{~s}$ maximal effort measurements, alternating between legs. A minimum pause of $60 \mathrm{~s}$ separated between trials and the highest of the six measurements was recorded [23]. Testing procedures, especially hip and knee joint angles were strictly enforced throughout the test. Also, to avoid patient discomfort, a thin foam was placed between the point of the application of the dynamometer and the skin of the patient's leg. Low muscle strength was defined as the relative KES of $\mathrm{KES} / \mathrm{BW}) \leq 0.34 \mathrm{~kg} / \mathrm{bw}$ for men and $\leq 0.24 \mathrm{~kg} / \mathrm{bw}$ for women (low scores were determined as 1 SD' below average scores found in a healthy older adult population, $n=700$ ) [34].

\section{Physical performance assessment 4-m gate speed}

Patients were asked to walk a 4-m distance at their regular pace. The tester used a hand-held stopwatch to record patients' completion times. Patients performed one orientation trial before performing two tests and the averaged time was computed. To covert test completion times to gate speed in meters/second $(\mathrm{m} / \mathrm{s})$, completion times were divided by 4 (meters). An impaired physical performance was considered a gait speed of $5 \mathrm{~s}$ or above corresponding to gait speed of $\leq 0.8 \mathrm{~m} / \mathrm{s}$ [16].

\section{Timed up and go test (TUG)}

Patients were seated in an armchair with a seat height of $\sim 44 \mathrm{~cm}$ and were timed on their ability to stand up from the chair, walk a 3-m course, turn safely around the cone, walk back, and sit down again (in seconds). The patients performed one preliminary trial before an actual test. A TUG test time of $\geq 20 \mathrm{~s}$ was indicative of impaired physical performance [16].

All muscle strength and physical performance assessments were performed by the same researcher throughout the study. Table 2 summarizes the EWGSOP1-2 guidelines defining cut-offs used to determine sarcopenia.

\section{Statistical analysis}

Study population characteristics are reported as mean values and standard deviations for continuous variables and percentages for categorical variables. Pearson correlations were calculated to evaluate the correlation between muscle mass (ASMI) and muscle strength (KES and HGS), as well as between muscle strength and measures of obesity (BMI, WC, body fat \%) (Table 3). A regression analysis using two blocks was conducted to examine which independent variable best predicts ASMI values. In the first block sex and weight were inserted as independent variables to control on the main characteristics of ASMI, using the enter method. The second block contained HGS, KES, vitamin D, HbA1c, performing physical activity, age, years of diabetes, and polypharmacy as independent variables to find which variable significantly influences ASMI after controlling for covariates, as well as the proportion of the predicted variance $\left(\mathrm{R}^{2}\right)$ of ASMI, explained by the independent variables. We used the stepwise method, where at each step, the independent variable with the highest effect on the dependent variable entered the equation (if its $p$-value was $<0.05$ ). The effect of these variables (including sex) on KES and HGS were examined in a multivariate analysis of covariance (MANCOVA). 
Table 2 EWGSOP1 vs EWGSOP2 sarcopenia cut-off values \& operational definitions

\begin{tabular}{|c|c|c|c|c|}
\hline \multicolumn{3}{|l|}{ EWGSOP1 Cut-off points } & \multicolumn{2}{|c|}{ EWGSOP2 cut-off points } \\
\hline Parameter & Men & Women & Men & Women \\
\hline Gait speed & $<0.8 \mathrm{~m} / \mathrm{s}$ & & $<0.8 \mathrm{~m} / \mathrm{s}$ & \\
\hline HGS & $<30 \mathrm{~kg}$ & $<20 \mathrm{~kg}$ & $<27 \mathrm{~kg}$ & $<16 \mathrm{~kg}$ \\
\hline ASMI & $<7.25 \mathrm{~kg} / \mathrm{m}^{2}$ & $<5.67 \mathrm{~kg} / \mathrm{m}^{2}$ & $<7 \mathrm{~kg} / \mathrm{m}^{2}$ & $<5.5 \mathrm{~kg} / \mathrm{m}^{2}$ \\
\hline \multicolumn{3}{|l|}{ EWGSOP1 definitions } & \multicolumn{2}{|c|}{ EWGSOP2 definitions } \\
\hline Pre/Probable Sarcopenia & \multicolumn{2}{|l|}{ Low ASMI } & \multicolumn{2}{|l|}{ Low HGS } \\
\hline Confirmed Sarcopenia & \multicolumn{2}{|c|}{ Low ASMI + Low HGS/ low Gait speed } & \multicolumn{2}{|c|}{ Low HGS + Low ASMI } \\
\hline Severe Sarcopenia & \multicolumn{2}{|c|}{ Low ASMI + Low HGS + low Gait speed } & \multicolumn{2}{|c|}{ Low HGS + Low ASMI + Low Gait speed } \\
\hline
\end{tabular}

Abbreviations: ASMI Appendicular Skeletal Mass Index, EWGSOP1 European Working Group on Sarcopenia in Older People 1, EWGSOP2 European Working Group on Sarcopenia in Older People 2, HGS Hand Grip Strength

\section{Results}

\section{Participant's characteristics}

Clinical characteristics

100 older patients with T2DM (40 men, 60 women) are listed in Table 1. Mean age was $70.5 \pm 4.6$ years, mean HbA1c was $7.47 \pm 1.14$ and BMI $31.7 \pm 5.95 \mathrm{~kg} / \mathrm{m}^{2}$. The prevalence of overweight (BMI $25.0-29.99 \mathrm{~kg} / \mathrm{m}^{2}$ ) and obesity (BMI $>30 \mathrm{~kg} / \mathrm{m}^{2}$ ) in our cohort was 92.5 and $65 \%$ in men, and 88 and $55 \%$ in women, respectively. The prevalence of central obesity as defined by waist circumference $(m e n \geq 102 \mathrm{~cm}$ and women $\geq 88 \mathrm{~cm}$ ) was observed in 87.5 and $96.4 \%$ of men and women, respectively.

\section{Muscle and functional characteristics}

Main mean values were (men vs women): ASMI (8.55 \pm 0.9 vs $\left.6.90 \pm 0.9 \mathrm{~kg} / \mathrm{m}^{2}\right)$, KES $(37.58 \pm 8.0 \mathrm{Kg}$ vs $24.0 \pm 5.6$ $\mathrm{Kg})$ and HGS $(40.06 \pm 6.83$ vs $24.97 \pm 4.3 \mathrm{Kg})$. The prevalence of low skeletal mass defined by EWGSOP2's ASMI cut-off points for low muscle mass was $7.5 \%$ in men and

Table 3 Correlations table (between sarcopenia parameters and anthropometric measurements)

\begin{tabular}{lll}
\hline Variable & Men & Women \\
\hline KES / ASMI & $r=0.55, p<0.001$ & $r=0.55, p<0.001$ \\
HGS / ASMI & $r=0.058, p=0.721$ & $r=0.14, p=0.157$ \\
KES / BMI & $r=0.45, p=0.004$ & $r=0.51, p<0.001$ \\
HGS / BMI & $r=0.133, p=0.41$ & $r=0.03, p=0.82$ \\
KES / body weight & $r=0.414, p=0.007$ & $r=0.5, p<0.001$ \\
HGS / body weight & $r=0.0, p=0.97$ & $r=0.038, p=0.814$ \\
KES / body fat \% & $r=0.22, p=0.19$ & $r=0.37, p=0.004$ \\
HGS / body fat \% & $r=-0.26, p=0.1$ & $r=-0.151, p=0.25$ \\
KES / WC & $r=0.32, p=0.049$ & $r=0.38, p=0.002$ \\
HGS / WC & $r=-0.17, p=0.27$ & $r=0.05, p=0.68$ \\
KES / height & $r=0.05, p=0.74$ & $r=-0.05, p=0.69$ \\
HGS / height & $r=0.36, p=0.02$ & $r=0.32, p=0.01$ \\
\hline Abbrevions: ASMIAppendicur Sket Mass, BMI Body Mass $1 n d e x, H G S$
\end{tabular}

Abbreviations: ASMI Appendicular Skeletal Mass, BMI Body Mass Index, HGS Hand Grip Strength, KES Knee Extension Strength, WC Waist Circumference
$10 \%$ in women. Slow gait speed was found in $7.5 \%$ of men $(n=3)$ and $6.7 \%$ of women $(n=4)$. Low TUG scores were found in $1.7 \%$ of women $(n=1)$ (see Table 1 for EWGSOP1 \&2 cut-off points).

Prevalence of probable and confirmed sarcopenia using HGS and KES cut-off points, low ASMI and low physical abilities (see Table 4 and Fig. 1 for prevalence of sarcopenia) Handgrip

According to EWGSOP2 Probable sarcopenia, defined by low HGS was identified in only one patient (woman). Confirmed sarcopenia by low HGS and low muscle mass, was not identified in any of our patients.

According to EWGSOP1 Pre-sarcopenia was identified in $10 \%$ of patients (three men and seven women). Sarcopenia, defined by low muscle mass and low HGS was identified in one patient (one woman).

\section{Knee extension}

According to EWGSOP2 Probable sarcopenia defined by relative KES was identified in $25 \%$ of patients (13 men and 12 women). Confirmed sarcopenic was identified in 3\% of patients (two men and one woman). Severe sarcopenia was identified in one patient (man).

\section{According to EWGSOP1}

Pre-sarcopenia, defined by low muscle mass was identified in $10 \%$ of patients (three men and seven women). Sarcopenia defined by low muscle mass and low relative KES was identified in $4 \%$ of patients (two men and two women). Severe sarcopenia was identified in one patient (man).

\section{Physical performance}

The prevalence of patients with low physical performance (gait speed) concurrently possessing low relative 
Table 4 Sarcopenia prevalence, status, and parameters, according to EWGSOP1 and EWGSOP1 definitions

\begin{tabular}{lll}
\hline Sarcopenia status & Sarcopenia parameter & EWGSOP1 \\
\hline Probable & Low ASMI & 3 men, 7 women (3, 7\%) \\
Confirmed & Low ASMI + Low HGS & 1 woman (1\%) \\
Confirmed & Low ASMI + Low KES & 2 men, 2 women $(2,2 \%)$ \\
Sarcopenia status & Sarcopenia parameter & EWGSOP2 \\
Pre sarcopenia & Low HGS & none \\
Pre sarcopenia & Low KES & 13 men, 12 women (13, 12\%)
\end{tabular}

Abbreviations: ASMI Appendicular Skeletal Mass Index, EWGSOP1 European Working Group on Sarcopenia in Older People 1, EWGSOP2 European Working Group on Sarcopenia in Older People 2, HGS Hand Grip Strength, KES Knee Extension Strength

KES was found in $66 \%(n=3)$ and $75 \%(n=4)$ of men and women respectively.

\section{Correlations of KES and HGS to ASMI Pearson correlation coefficients}

KES scores were found to have a moderate correlation to ASMI in men $(r=0.551 p<0.001, n=38)$, (Fig. 2a) and women $(r=0.551, p<0.001, n=60)$, (Fig. 2b). Conversely, HGS was not found to correlate to ASMI in either men $(r=0.058, p=0.721, n=40)$ (Fig. 2c) nor women ( $r=0.139, p=0.157, n=60)$, (Fig. 2d). Also, KES but not HGS was found to have a moderate correlation with BMI $(r=0.45, p=0.004 ; r=0.51, p<0.001)$ and a weak correlation with BW $(r=0.41, p=0.007$; $r=0.5, p<0.001)$ and WC $(r=0.32, p=0.049 ; r=0.38$, $p<0.002)$ in men and women respectively. Body fat $\%$ had a weak correlation only to KES and only in women $(r=0.37, p<0.001)$. HGS was found to possess a weak correlation to body weight in the total cohort $(r=0.41, p<0.001)$. Height showed a weak correlation to HGS but not to KES in both men $(r=$ $0.36, p=0.02)$ and women $(r=0.32, p=0.01)$, respectively (Table 3). (Interpretation of the Pearson's correlation coefficients: 0.00 to 0.25 very weak; 0.26 to 0.49 weak; 0.50 to 0.69 moderate; 0.70 to 0.89 strong; 0.90 to 1.00 very strong) [36].

\section{Multivariate analysis}

We conducted a linear regression analysis with two blocks to predict the ASMI. In the first block sex and weight were inserted as independent variables to control on the main characteristics of the ASMI, using the enter

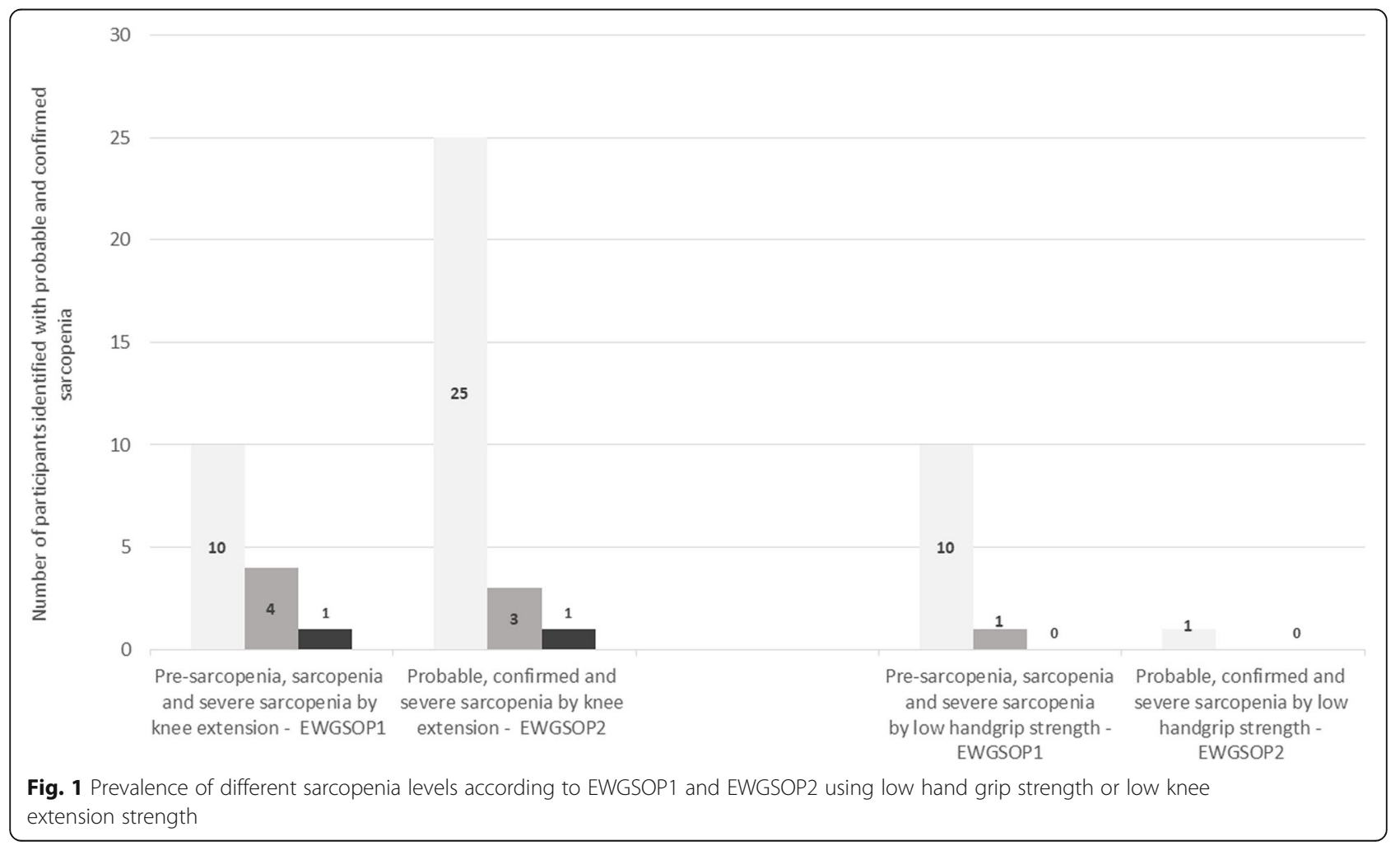




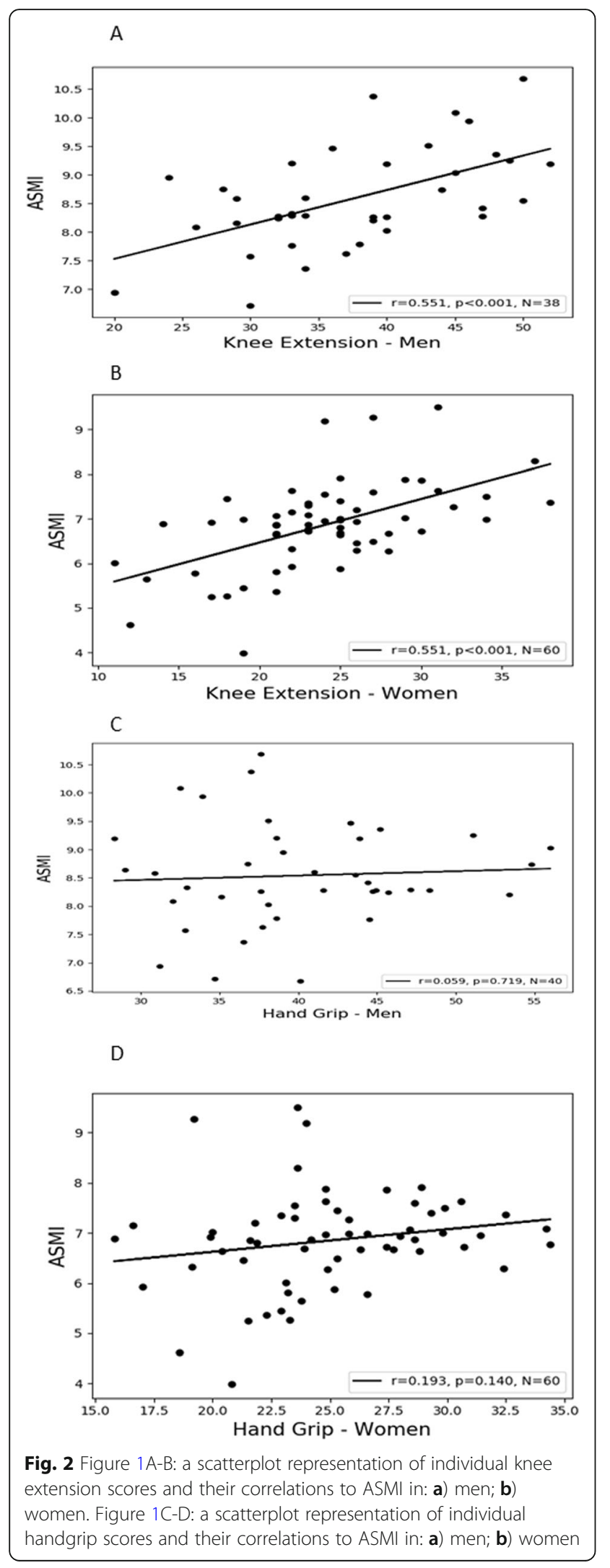

method. In the second block the stepwise method was used, containing HGS, KES, vitamin D levels, HbA1c, conducting physical activity, age, duration of T2DM and number of medications as independent variables to find which variable significantly influences ASMI after controlling for the covariates (sex and weight). At each step, the independent variable not in the equation possessing the smallest probability of $\mathrm{F}$ was entered, if its $p$-value in the model was $<0.05$. In the first block, regression analysis revealed that $87.8 \%$ of the predicted variance $\left(R^{2}\right)$ of the ASMI was explained by sex and weight. In the second block, KES was the only variable that entered the model, meaning that after clearing the common variance with sex and weight, HGS (beta in $=0.053, p=0.459$ ), vitamin $\mathrm{D}$ levels (beta in $=-0.034, p=0.373$ ), HbA1c (beta in $=-0.006, p=0.875$ ), physical activity (beta in = $-0.063, p=0.091$ ), age (beta in $=0.042, p=0.281$ ), duration of T2DM (beta in $=-0.008, p=0.858$ ), and polypharmacy (beta in $=-0.011, p=0.770$ ) were not correlated with ASMI. The final model revealed that $88.2 \%$ of the predicted variance $\left(R^{2}\right)$ of ASMI could be explained by weight, sex, and KES $(p<0.001)$ (Table 5$)$. The correlation matrix with regression variables can be found in supplementary files.

\section{The effect of independent variables on knee extension strength and handgrip strength}

A MANCOVA test was used to examine the association between groups of high and low values of weight $(<$ mean weight), age $(<75), \mathrm{HbA1c}(<7.5)$, years with T2DM $(<$ $10)$, Vitamin D levels $(<25)$, polypharmacy $(\geq 8)$ and physical activity (yes / no), as independent variables with sex as covariant, to KES and HGS as dependent variables (Table 6). There was a statistically significant difference in KES and HGS based on the weight and years with T2DM. Univariate ANOVAs revealed weight was only associated with KES $(\mathrm{F}=6.59, p<0.05)$, but not with HGS ( $F=0.009$, NS). Meaning, participants with higher weight had higher KES. Moreover, ANOVAs indicated that both KES and HGS were significantly correlated to duration of T2DM $(p<0.01),(p<0.05)$. Participants with less than 10 years of diabetes had greater KES and HGS.

\section{Discussion}

The main finding of our study was that cut-off points for low KES identified considerably more patients with probable and confirmed sarcopenia compared to HGS testing using the EWGSOP2 cut-off points for low HGS. The low prevalence of sarcopenia using HGS cut-off points in older adults with T2DM found in our study (3\%) was also observed by Villani et al. [37] identifying $2.3 \%$ of his T2DM older patients with confirmed sarcopenia (age eligibility $\geq 50$ years) and Freitas's et al. [38] identifying a somewhat higher prevalence rate of 
Table 5 Hierarchical multiple regression

\begin{tabular}{|c|c|c|c|c|c|c|c|}
\hline Block & Predicting Variable & $\mathbf{t}$ & $\beta$ & F change & $\mathrm{R}^{2}$ change & $\mathrm{F}$ & $R^{2}$ \\
\hline \multirow[t]{2}{*}{1} & Weight & 14.127 & $.701^{* * *}$ & & & $310.351^{* * * *}$ & 0.878 \\
\hline & Sex & -4.272 & $-.231^{* * *}$ & & & & \\
\hline 2 & Knee extension strenght & 2.012 & $.124^{*}$ & $4.048^{*}$ & 0.005 & $215.756^{* * *}$ & 0.882 \\
\hline
\end{tabular}

Parameters in regression model ${ }^{*} p<0.05,{ }^{* *} p<0.01,{ }^{* * *} P<0.001$

Note: $t$ - The $t$ statistic is the coefficient divided by its standard error. $\beta$ is the normalized coefficient, the highest its absolute value, the variable have more influence on the depended variable. The $\mathrm{F}$ value in regression is the result of a test where the null hypothesis is that all of the regression coefficients are equal to zero. $R^{2}$ - coefficient of determination is a statistical measure of how well the regression predictions approximate the real data points

confirmed sarcopenia (7\%) and mostly women (88\%), potentially due to a higher ASMI cut-off point for women $(6.0 \mathrm{~kg} / \mathrm{m} 2)$. This observation is perplexing since T2DM older patients are known to possess markedly reduced muscle strength [5], and in particular low handgrip strength [39], with an increased risk for sarcopenia $[40,41]$.

Despite the EWGSOP2 algorithm's potential to reduce health costs by reducing the number of DXA measurements to identify sarcopenic patients [42], it has been often scrutinized by investigators, observing a markedly lower prevalence of probable and confirmed sarcopenia compared to the earlier EWGSOP1 guidelines. This lower prevalence of sarcopenia is mainly due to the reduced cut-off points for both HGS ( $3 \mathrm{k}$ "g and a $4 \mathrm{k}$ "g reduction in HGS for men and women respectively), and ASMI $\left(\right.$ a $0.25 \mathrm{~kg} / \mathrm{m}^{2}$ and $0.17 \mathrm{~kg} / \mathrm{m}^{2}$ reductions in men and women respectively [42-45]. Indeed, in our cohort of older T2DM patients, a higher prevalence of mostly pre-sarcopenia (10\%) and confirmed sarcopenia (4\%) was identified using the EWGSOP1 cut-off points for low HGS.

The EWGSOP2 recommends the use of one or two strength tests as their primary criterion for the identification of sarcopenia, an upper body test (HGS) and a lower body test (five-repetition chair stands) [16]. We

Table 6 Multivariate Analysis of Covariance (MANCOVA) effects on knee extension and handgrip strength

\begin{tabular}{lllll}
\hline Variable & Wilks' $\boldsymbol{\lambda}$ & $\mathbf{f}$ & DF/DF Error & $\begin{array}{l}\text { Partial } \\
\mathbf{n 2}\end{array}$ \\
\hline Weight & 0.915 & $3.562^{*}$ & 2,77 & 0.085 \\
Age & 0.945 & 2.221 & 2,77 & 0.055 \\
T2DM Years & 0.887 & $4.925^{*}$ & 2,77 & 0.113 \\
HbA1C & 0.988 & 0.462 & 2,77 & 0.012 \\
Vitamin D & 0.987 & 0.512 & 2,77 & 0.013 \\
\# of drugs & 0.998 & 0.077 & 2,77 & 0.002 \\
Physical Act & 0.987 & 0.492 & 2,77 & 0.013 \\
\hline
\end{tabular}

${ }^{*} p<0.05$

Note: Wilks' $\lambda$ represents the ratio between the error variance (or covariance) and the effect variance (or covariance), $\mathrm{F}$ is the statistics, DF - degree of freedom, Partial $\eta 2$ - effect size, represents the proportion of the variance in the dependent variable that can be explained by the variance in the groups of a categorical independent variable believe that the addition of a lower body strength test to the HGS test is imperative since the HGS is a poor predictor of both total body strength [20], and functional performance $[46,47]$, while yielding dissimilar sarcopenia rates compared to lower body testing (chair stands), in a community-dwelling group of middle aged and older adults [48]. The KES test can be recommended as an alternative lower body strength test for the chair rise test, as it involves less complex weight-bearing body movements [49], is better suited for diabetics with peripheral neuropathy [50], as well as obese older adults with moderate to advanced osteoarthritis [51, 52].

Our study was not exclusive in observing that the identification of probable sarcopenia is dependent on the strength tests performed. Wearing et al. has shown that by choosing a proper strength screening test $27 \%$ of patients would benefit from the continuation of the screening process, possibly preventing further strength reductions through an early initiation of suitable interventions [29]. The higher prevalence of probable sarcopenia in measures other than HGS was found in other studies using the new EWGSOP2 algorithm. Higher rates of probable sarcopenia using the chair rise test were found by Kim et (13\% in women) [53], as well as Johansson et al. (4.4\% vs $1.3 \%$ ) [48], noticing that subjects identified with probable sarcopenia by chair raise, were heavier and more obese than subjects identified by HGS, probably due to the greater influence of relative leg muscle strength. Dodd et al. observed that chair rise detected double the prevalence rates of probable sarcopenia in comparison to the HGS (15\% vs $7 \%)$, stating the need to perform both tests to better assess probable sarcopenia [54].

The correlation found between KES and ASMI along with other body size measures (BMI, body weight, WC) is important as it can be associated with an "obesity paradox". The obesity paradox is generally referring to the protective effect obesity imparts on decreased mortality in older adults [55]. That said, the term obesity paradox has also been related to the added muscle mass $[11,56]$ and muscle strength [57], Caused by the anabolic effect that occurs through continuously carrying the added body mass associated with obesity. This phenomenon is especially expressed in the lower body 
[58], through an increase in absolute KES [59], underscoring the need to normalize KES to body size using relative strength cut-off points. Relative muscle strength (muscle strength divided by bodyweight) better identifies individuals with reduced muscle strength through either HGS $[60,61]$ or KES testing $[28,62]$, thus possibly reducing false negative sarcopenia assessments.

The high prevalence of obesity in our cohort, can possibly explain the low prevalence of confirmed sarcopenia through low ASMI scores that coincide with the higher BMI values generally associated with higher fat free mass [63].

Overall, the prevalence of common health complications exhibited by the subjects in our study was somewhat lower than values generally seen in older T2DM patients [64]. Our finding that muscle strength (both HGS and KES) was significantly correlated to duration of T2DM is in line with chen et al. [62], while unlike Izzo et al. [14], stating that diabetes duration does not increase the prevalence of sarcopenia. The finding that most of the patients possessing low physical performance scores, concurrently possess low relative KES compared to those with impaired HGS, further affirms the added value in using KES as a sarcopenia screening tool.

Our study has several limitations. Its main limitation is the relatively small sample of T2DM patients making our findings difficult to generalize to older adults with T2DM. Additionally, the majority of our patients were younger than 75 years of age with only a small number of patients being older than 75 , thus reducing the potential to truly investigate the age factor on outcome measures.

\section{Conclusion}

In a cohort of 100 mostly obese T2DM older patients, relative KES cut-off points using a simple hand-held dynamometer can assist in the identification of mostly probable sarcopenia and confirmed sarcopenia cases using EWGSOP2 cut off points for low muscle mass. Using the EWGSOP2 cut off points for low muscle strength by HGS mostly failed to identify probable and confirmed sarcopenia, possibly due to high prevalence of normal absolute handgrip and ASMI values associated with subjects possessing high BMI and body weight.

It must be stated that our study is the first to assess the prevalence of probable and confirmed sarcopenia in older T2DM patients by relative KES cut off points. Due to the importance of the initial screening strength test for further analysis and early treatment of sarcopenia and to reduce false negative sarcopenia, it would be prudent to add a lower body strength test such as the relative KES while screening older T2DM patients for sarcopenia while using the EWGSOP2 guidelines.

\section{Abbreviations}

ANOVA: one-way analysis of variance; ASMI: appendicular skeletal mass index; BIA: bioelectrical impendence analysis; BMI: body mass index; EWGSOP 1/2: The European Working Group on Sarcopenia in Older People; HGS: handgrip strength; KES: knee extension strength; MANCOVA: multivariate analysis of covariance; T2DM: Type 2 Diabetes Mellitus; TUG: timed up and go test; WC: Waist circumference

\section{Acknowledgements}

This work was performed in partial fulfillment of the requirements for a Ph.D. degree by Ofer Kis at the Faculty of Health Sciences, Ariel University, Ariel, Israel.

\section{Authors' contributions}

$O K, A B$ and $A R$ conceived the study; OK, $A B, R E, A D, N S$ and DSM designed the study; $O K$ and $A B$ analyzed the data; $O K$ and $A B$ wrote the manuscript; $A D, N S, R E$ and DSM reviewed the manuscript. All authors read and approved the final manuscript.

\section{Funding}

None.

\section{Availability of data and materials}

The datasets generated and/or analyzed during the current study are not publicly available due to institutional restrictions but are available from the corresponding author on reasonable request.

\section{Declarations}

Ethics approval and consent to participate

The study has received ethics approval from the Tel-Aviv Sourasky Medical Center Institutional Review Board. All participants will provide written informed consent to participate in the study.

\section{Consent for publication}

All authors provided consent for publication.

\section{Competing interests}

The authors declare that they have no competing interests.

\section{Author details}

${ }^{1}$ The Faculty of Health Sciences, Ariel University, Ariel, Israel. ${ }^{2}$ Institute of Endocrinology, Metabolism and Hypertension, Tel Aviv Sourasky Medical Center, 6 Weizmann St, 64239 Tel-Aviv, Israel. ${ }^{3}$ The Sagol Center for Epigenetics of Metabolism and Aging, Tel Aviv Sourasky Medical Center, Tel-Aviv, Israel. ${ }^{4}$ School of Health Sciences, Ashkelon Academic College, Ashkelon, Israel. ${ }^{5}$ The Academic College at Wingate, Wingate Institute, Netanya, Israel. ${ }^{6}$ The Sackler Faculty of Medicine Tel-Aviv University, Tel-Aviv, Israel.

Received: 20 May 2021 Accepted: 25 November 2021

Published online: 27 January 2022

\section{References}

1. Centers for Disease Control and Prevention. National Diabetes Statistics Report, 2020. Atlanta, GA: Centers for Disease Control and Prevention, U.S. Dept of Health and Human Services; 2020.

2. Key global findings 2021. The IDF Diabetes Atlas 10th Edition. (02021 International Diabetes Federation. https://www.diabetes.org/.

3. Umegaki H. Sarcopenia and frailty in older patients with diabetes mellitus. Geriatr Gerontol Int. 2016;16(3):293-9. https://doi.org/10.1111/ggi.12688.

4. Kalyani RR, Tra Y, Yeh H-C, Egan JM, Ferrucci L, Brancati FL. Quadriceps strength, quadriceps power, and gait speed in older U.S. adults with diabetes: results from the National Health and nutrition examination survey (NHANES), 1999-2002. J Am Geriatr Soc. 2013;61(5):769-75. https://doi.org/1 $0.1111 /$ jgs. 12204 .

5. Leenders $M$, Verdijk LB, van der Hoeven L, Adam JJ, van Kranenburg J, Nilwik $R$, et al. Patients with type 2 diabetes show a greater decline in muscle mass, muscle strength, and functional capacity with aging. J Am Med Dir Assoc. 2013;14(8):585-92. https://doi.org/10.1016/j.jamda.2 013.02.006. 
6. Wong E, Backholer K, Gearon E, Harding J, Freak-Poli R, Stevenson C, et al. Diabetes and risk of physical disability in adults: a systematic review and meta-analysis. Lancet Diabetes Endocrinol. 2013;1(2):106-14. https://doi. org/10.1016/S2213-8587(13)70046-9.

7. Calvani R, Rodriguez-Mañas L, Picca A, Marini F, Biancolillo A, Laosa O, et al The "metabolic biomarkers of frailty in older people with type 2 diabetes mellitus" (MetaboFrail) study: rationale, design and methods. Exp Gerontol. 2020;129:110782. https://doi.org/10.1016/j.exger.2019.110782.

8. Corriere M, Rooparinesingh N, Kalyani RR. Epidemiology of Diabetes and Diabetes Complications in the Elderly: An Emerging Public Health Burden. Curr Diabetes Rep. 2013;13:805-13 Available from: https://www.ncbi.nlm.nih. gov/pmc/articles/PMC3856245/.

9. Cruz-Jentoft AJ, Baeyens JP, Bauer JM, Boirie Y, Cederholm T, Landi F, et al. Sarcopenia: European consensus on definition and diagnosis. Age Ageing. 2010;39(4):412-23. https://doi.org/10.1093/ageing/afq034.

10. Cui M, Gang X, Wang G, Xiao X, Li Z, Jiang Z, Wang G. A cross-sectional study: Associations between sarcopenia and clinical characteristics of patients with type 2 diabetes. Medicine (Baltimore). 2020;99(2):e18708.

11. Mesinovic J, McMillan LB, Shore-Lorenti C, De Courten B, Ebeling PR, Scott D. Metabolic Syndrome and Its Associations with Components of Sarcopenia in Overweight and Obese Older Adults. J Clin Med. 2019; 8(2):145.

12. Murata Y, Kadoya Y, Yamada S, Sanke T. Sarcopenia in elderly patients with type 2 diabetes mellitus: prevalence and related clinical factors. Diabetol Int. 2017;9(2):136-42. https://doi.org/10.1007/s13340-017-0339-6.

13. Sinclair AJ, Abdelhafiz AH, Rodríguez-Mañas L. Frailty and sarcopenia - newly emerging and high impact complications of diabetes. J Diabetes Complicat. 2017;31(9):1465-73. https://doi.org/10.1016/j.jdiacomp.2017.05.003.

14. Izzo A, Massimino E, Riccardi G, Della Pepa G. A Narrative Review on Sarcopenia in Type 2 Diabetes Mellitus: Prevalence and Associated Factors. Nutrients. 2021;13(1):183

15. Mayhew AJ, Amog K, Phillips S, Parise G, McNicholas PD, de Souza RJ, et al. The prevalence of sarcopenia in community-dwelling older adults, an exploration of differences between studies and within definitions: a systematic review and meta-analyses. Age Ageing. 2019;48(1):48-56. https:// doi.org/10.1093/ageing/afy106.

16. Cruz-Jentoft AJ, Bahat $G$, Bauer J, Boirie $Y$, Bruyère $O$, Cederholm T, et al. Sarcopenia: revised European consensus on definition and diagnosis. Age Ageing. 2019;48(1):16-31. https://doi.org/10.1093/ageing/afy169.

17. Samuel D, Rowe P, Hood V, Nicol A. The relationships between muscle strength, biomechanical functional moments and health-related quality of life in non-elite older adults. Age Ageing. 2012;41(2):224-30. https://doi. org/10.1093/ageing/afr156

18. Park SW, Goodpaster BH, Strotmeyer ES, Kuller LH, Broudeau R, Kammerer $C$, et al. Accelerated loss of skeletal muscle strength in older adults with type 2 diabetes: the health, aging, and body composition study. Diabetes Care Am Diabetes Assoc. 2007:30(6):1507-12. https://doi. org/10.2337/dc06-2537.

19. Andersen $\mathrm{H}$, Nielsen $\mathrm{S}$, Mogensen $\mathrm{CE}$, Jakobsen J. Muscle strength in type 2 diabetes. Diabetes. 2004;53(6):1543-8. https://doi.org/10.2337/dia betes.53.6.1543.

20. Yeung SSY, Reijnierse EM, Trappenburg MC, Hogrel J-Y, McPhee JS, Piasecki $M$, et al. Handgrip strength cannot be assumed a proxy for overall muscle strength. J Am Med Dir Assoc. 2018;19(8):703-9. https://doi.org/10.1016/j.ja mda.2018.04.019.

21. IJzerman TH, Schaper NC, Melai T, Meijer K, Willems PJB, Savelberg HHCM. Lower extremity muscle strength is reduced in people with type 2 diabetes, with and without polyneuropathy, and is associated with impaired mobility and reduced quality of life. Diabetes Res Clin Pract. 2012;95(3):345-51. https://doi.org/10.1016/j.diabres.2011.10.026.

22. Buckinx F, Croisier J-L, Charles A, Petermans J, Reginster J-Y, Rygaert $X$, et al. Normative data for isometric strength of 8 different muscle groups and their usefulness as a predictor of loss of autonomy among physically active nursing home residents: the SENIOR cohort. J Musculoskelet Neuronal Interact. 2019;19(3):258-65.

23. Muff G, Dufour S, Meyer A, Severac F, Favret F, Geny B, et al. Comparative assessment of knee extensor and flexor muscle strength measured using a hand-held vs. isokinetic dynamometer. J Phys Ther Sci. 2016;28(9):2445-51. https://doi.org/10.1589/jpts.28.2445.

24. Mentiplay BF, Perraton LG, Bower KJ, Adair B, Pua Y-H, Williams GP, et al. Assessment of lower limb muscle strength and power using hand-held and fixed dynamometry: a reliability and validity study. PLoS One. 2015;10(10): e0140822. https://doi.org/10.1371/journal.pone.0140822.

25. Chamorro C, Armijo-Olivo S, De la Fuente C, Fuentes J, Javier CL. Absolute reliability and concurrent validity of hand held dynamometry and isokinetic dynamometry in the hip, knee and ankle joint: systematic review and metaanalysis. Open Med (Wars). 2017;12(1):359-75. https://doi.org/10.1515/med-2 017-0052.

26. Buckinx F, Croisier J-L, Reginster J-Y, Dardenne N, Beaudart C, Slomian J, et al. Reliability of muscle strength measures obtained with a hand-held dynamometer in an elderly population. Clin Physiol Funct Imaging. 2017; 37(3):332-40. https://doi.org/10.1111/cpf.12300.

27. Mori H, Kuroda A, Matsuhisa M. Clinical impact of sarcopenia and dynapenia on diabetes. Diabetol Int. 2019;10(3):183-7. https://doi.org/10.1007/s13340019-00400-1.

28. Choquette S, Bouchard DR, Doyon CY, Sénéchal M, Brochu M, Dionne IJ. Relative strength as a determinant of mobility in elders 67-84 years of age. A nuage study: nutrition as a determinant of successful aging. J Nutr Health Aging. 2010;14(3):190-5. https://doi.org/10.1007/s12603-010-0047-4.

29. Wearing J, Konings $P$, de Bie RA, Stokes M, de Bruin ED. Prevalence of probable sarcopenia in community-dwelling older Swiss people - a cross-sectional study. BMC Geriatr. 2020;20(1):307. https://doi.org/10.1186/s12877-020-01718-1.

30. Buch A, Eldor R, Kis O, Keinan-Boker L, Dunsky A, Rubin A, et al. The effect of circuit resistance training, empagliflozin or "vegeterranean diet" on physical and metabolic function in older subjects with type 2 diabetes: a study protocol for a randomized control trial (CEV-65 trial). BMC Geriatr. 2019:19(1):228. https://doi.org/10.1186/s12877-019-1219-7.

31. Ling CHY, de Craen AJM, Slagboom PE, Gunn DA, Stokkel MPM, Westendorp RGJ, et al. Accuracy of direct segmental multi-frequency bioimpedance analysis in the assessment of total body and segmental body composition in middle-aged adult population. Clin Nutr. 2011;30(5):610-5. https://doi.org/10.1016/j.clnu.2011.04.001.

32. Fried LP, Tangen CM, Walston J, Newman AB, Hirsch C, Gottdiener J, et al. Frailty in older adults evidence for a phenotype. J Gerontol A Biol Sci Med Sci. 2001;56(3):M146-57. https://doi.org/10.1093/gerona/56.3.M146.

33. Bohannon RW, Wikholm JB. Measurements of knee extension force obtained by two examiners of substantially different experience with a hand-held dynamometer. Isokinet Exerc Sci IOS Press. 1992;2(1):5-8. https:// doi.org/10.3233/IES-1992-2101.

34. Martien S, Delecluse C, Boen F, Seghers J, Pelssers J, Van Hoecke A-S, et al. Is knee extension strength a better predictor of functional performance than handgrip strength among older adults in three different settings. Arch Gerontol Geriatr. 2015;60(2):252-8. https://doi.org/10.1016/j.archger.2014.11.010

35. Chumlea WC, Guo SS, Kuczmarski RJ, Flegal KM, Johnson CL, Heymsfield SB, et al. Body composition estimates from NHANES III bioelectrical impedance data. Int J Obes Relat Metab Disord. 2002;26(12):1596-609.

36. Domholdt E. Physical therapy research: principles and applications. 2nd ed. Philadelophia: WB Saunders Co.; 2000

37. Villani A, McClure R, Barrett M, Scott D. Diagnostic differences and agreement between the original and revised European working group (EWGSOP) consensus definition for sarcopenia in community-dwelling older adults with type 2 diabetes mellitus. Arch Gerontol Geriatr. 2020;89:104081. https://doi.org/10.1016/j.archger.2020.104081.

38. de Freitas MM, de Oliveira VLP, Grassi T, Valduga K, Miller MEP, Schuchmann RA, et al. Difference in sarcopenia prevalence and associated factors according to 2010 and 2018 European consensus (EWGSOP) in elderly patients with type 2 diabetes mellitus. Exp Gerontol. 2020;132:110835. https://doi.org/10.1016/j.exger.2020.110835.

39. Anagnostis P, Gkekas NK, Achilla C, Pananastasiou G, Taouxidou P, Mitsiou $M$, et al. Type 2 diabetes mellitus is associated with increased risk of sarcopenia: a systematic review and Meta-analysis. Calcif Tissue Int. 2020; 107(5):453-63. https://doi.org/10.1007/s00223-020-00742-y.

40. Chen F, Xu S, Wang Y, Chen F, Cao L, Liu T, et al. Risk factors for sarcopenia in the elderly with type 2 diabetes mellitus and the effect of metformin. J Diabetes Res. 2020;2020:3950404-10. https://doi.org/10.1155/2020/3950404.

41. Wang T, Feng X, Zhou J, Gong H, Xia S, Wei Q, Hu X, Tao R, Li L, Qian F, Yu L. Type 2 diabetes mellitus is associated with increased risks of sarcopenia and pre-sarcopenia in Chinese elderly. Sci Rep. 2016;6:38937.

42. Reiss J, Iglseder B, Alzner R, Mayr-Pirker B, Pirich C, Kässmann H, et al. Consequences of applying the new EWGSOP2 guideline instead of the former EWGSOP guideline for sarcopenia case finding in older patients. Age Ageing. 2019;48(5):719-24. https://doi.org/10.1093/ageing/afz035. 
43. Petermann-Rocha F, Chen M, Gray SR, Ho FK, Pell JP, Celis-Morales C. New versus old guidelines for sarcopenia classification: what is the impact on prevalence and health outcomes. Age Ageing. 2020;49(2):300-4. https://doi. org/10.1093/ageing/afz126.

44. Locquet $M$, Beaudart $C$, Petermans J, Reginster J-Y, Bruyère O. EWGSOP2 versus EWGSOP1: impact on the prevalence of sarcopenia and its major health consequences. J Am Med Dir Assoc. 2019;20(3):384-5. https://doi. org/10.1016/j.jamda.2018.11.027.

45. Costanzo L, De Vincentis A, Di lorio A, Bandinelli S, Ferrucci L, Antonelli Incalzi $R$, et al. Impact of low muscle mass and low muscle strength according to EWGSOP2 and EWGSOP1 in community-dwelling older people. J Gerontol A Biol Sci Med Sci. 2020;75(7):1324-30. https://doi.org/1 0.1093/gerona/glaa063.

46. Harris-Love MO, Benson K, Leasure E, Adams B, Mclntosh V. The influence of upper and lower extremity strength on performance-based sarcopenia assessment tests. J Funct Morphol Kinesiol. 2018;3(4). https://doi.org/10.33 90/jfmk3040053.

47. Yee XS, Ng YS, Allen JC, Latib A, Tay EL, Abu Bakar HM, et al. Performance on sit-to-stand tests in relation to measures of functional fitness and sarcopenia diagnosis in community-dwelling older adults. Eur Rev Aging Phys Act. 2021;18(1):1. https://doi.org/10.1186/s11556-020-00255-5.

48. Johansson J, Strand BH, Morseth B, Hopstock LA, Grimsgaard S. Differences in sarcopenia prevalence between upper-body and lower-body based EWGSOP2 muscle strength criteria: the Tromsø study 2015-2016. BMC Geriatr. 2020;20(1):461. https://doi.org/10.1186/s12877-020-01860-w.

49. Yokoyama H, Shiraiwa T, Takahara M, Iwamoto M, Kuribayashi N, Nomura T, et al. Applications of physical performance measures to routine diabetes care for frailty prevention concept: fundamental data with grip strength, gait speed, timed chair stand speed, standing balance, and knee extension strength. BMJ Open Diabetes Res Care. 2020;8(1):e001562. https://doi.org/1 0.1136/bmjdrc-2020-001562.

50. Riandini T, Wee HL, Khoo EYH, Tai BC, Wang W, Koh GCH, et al. Functional status mediates the association between peripheral neuropathy and healthrelated quality of life in individuals with diabetes. Acta Diabetol. 2018;55(2): 155-64. https://doi.org/10.1007/s00592-017-1077-8.

51. Koblbauer IFH, Lambrecht $Y$, van der Hulst MLM, Neeter C, Engelbert RHH, Poolman RW, et al. Reliability of maximal isometric knee strength testing with modified hand-held dynamometry in patients awaiting total knee arthroplasty: useful in research and individual patient settings? A reliability study. BMC Musculoskelet Disord. 2011;12(1):249. https://doi.org/10.1186/14 71-2474-12-249.

52. Verlaan L, Boekesteijn RJ, Oomen PW, Liu W-Y, Peters MJM, Witlox MA, et al. Biomechanical alterations during sit-to-stand transfer are caused by a synergy between knee osteoarthritis and obesity. Biomed Res Int. 2018;2018: 3519498-7. https://doi.org/10.1155/2018/3519498.

53. Kim M, Won CW. Prevalence of sarcopenia in community-dwelling older adults using the definition of the European working group on sarcopenia in older people 2: findings from the Korean frailty and aging cohort study. Age Ageing. 2019;48(6):910-6. https://doi.org/10.1093/ageing/afz091.

54. Dodds RM, Murray JC, Robinson SM, Sayer AA. The identification of probable sarcopenia in early old age based on the SARC-F tool and clinical suspicion: findings from the 1946 British birth cohort. Eur Geriatr Med. 2020; 11(3):433-41. https://doi.org/10.1007/s41999-020-00310-5.

55. Gruberg L, Weissman NJ, Waksman R, Fuchs S, Deible R, Pinnow EE, et al. The impact of obesity on the short-term and long-term outcomes after percutaneous coronary intervention: the obesity paradox? J Am Coll Cardiol. 2002;39(4):578-84. https://doi.org/10.1016/S0735-1097(01)01802-2.

56. Smeuninx B, Mckendry J, Wilson D, Martin U, Breen L. Age-related anabolic resistance of Myofibrillar protein synthesis is exacerbated in obese inactive individuals. J Clin Endocrinol Metab. 2017;102(9):3535-45. https://doi.org/1 0.1210/jc.2017-00869.

57. Tomlinson DJ, Erskine RM, Morse Cl, Winwood K, Onambélé-Pearson G. The impact of obesity on skeletal muscle strength and structure through adolescence to old age. Biogerontology. 2016;17(3):467-83. https://doi.org/1 0.1007/s10522-015-9626-4.

58. Morgan PT, Smeuninx B, Breen L. Exploring the impact of obesity on skeletal muscle function in older age. Front Nutr. 2020;7:569904. https://doi. org/10.3389/fnut.2020.569904.

59. Koushyar H, Nussbaum MA, Davy KP, Madigan ML. Relative strength at the hip, knee, and ankle is lower among younger and older females who are obese. J Geriatr Phys Ther. 2017;40(3):143-9. https://doi.org/10.1519/JPT. 0000000000000086.

60. Lee W-J, Peng L-N, Chiou S-T, Chen L-K. Relative handgrip strength is a simple Indicator of Cardiometabolic risk among middle-aged and older people: a nationwide population-based study in Taiwan. PLoS One. 2016; 11(8):e0160876. https://doi.org/10.1371/journal.pone.0160876.

61. Lombardo M, Padua E, Campoli F, Panzarino M, Mîndrescu V, Annino G, et al. Relative handgrip strength is inversely associated with the presence of type 2 diabetes in overweight elderly women with varying nutritional status. Acta Diabetol. 2021;58(1):25-32. https://doi.org/10.1007/s00592-02001588-4.

62. Chen C-N, Chen T-C, Tsai S-C, Hwu C-M. Factors associated with relative muscle strength in patients with type 2 diabetes mellitus. Arch Gerontol Geriatr. 2021;95:104384. https://doi.org/10.1016/j.archger.2021.104384.

63. Donini LM, Pinto A, Giusti AM, Lenzi A, Poggiogalle E. Obesity or BMI paradox? Beneath the tip of the iceberg. Front Nutr. 2020;7:53. https://doi. org/10.3389/fnut.2020.00053.

64. Iglay K, Hannachi H, Joseph Howie P, XU J, Li X, Engel SS, et al. Prevalence and co-prevalence of comorbidities among patients with type 2 diabetes mellitus. Curr Med Res Opin. 2016;32(7):1243-52. https://doi.org/10.1185/03 007995.2016.1168291.

\section{Publisher's Note}

Springer Nature remains neutral with regard to jurisdictional claims in published maps and institutional affiliations.
Ready to submit your research? Choose BMC and benefit from:

- fast, convenient online submission

- thorough peer review by experienced researchers in your field

- rapid publication on acceptance

- support for research data, including large and complex data types

- gold Open Access which fosters wider collaboration and increased citations

- maximum visibility for your research: over $100 \mathrm{M}$ website views per year

At BMC, research is always in progress.

Learn more biomedcentral.com/submissions 